\title{
Review Article \\ Epigenetic Reprogramming of Muscle Progenitors: Inspiration for Clinical Therapies
}

\author{
Silvia Consalvi, ${ }^{1}$ Martina Sandoná, ${ }^{1,2}$ and Valentina Saccone ${ }^{1}$ \\ ${ }^{1}$ IRCSS, Fondazione Santa Lucia, Via del Fosso di Fiorano 64, 00143 Roma, Italy \\ ${ }^{2}$ DAHFMO-Unit of Histology and Medical Embryology, University of Rome "La Sapienza", Rome, Italy
}

Correspondence should be addressed to Valentina Saccone; v.saccone@hsantalucia.it

Received 15 May 2015; Revised 14 September 2015; Accepted 16 September 2015

Academic Editor: James G. Ryall

Copyright (C) 2016 Silvia Consalvi et al. This is an open access article distributed under the Creative Commons Attribution License, which permits unrestricted use, distribution, and reproduction in any medium, provided the original work is properly cited.

\begin{abstract}
In the context of regenerative medicine, based on the potential of stem cells to restore diseased tissues, epigenetics is becoming a pivotal area of interest. Therapeutic interventions that promote tissue and organ regeneration have as primary objective the selective control of gene expression in adult stem cells. This requires a deep understanding of the epigenetic mechanisms controlling transcriptional programs in tissue progenitors. This review attempts to elucidate the principle epigenetic regulations responsible of stem cells differentiation. In particular we focus on the current understanding of the epigenetic networks that regulate differentiation of muscle progenitors by the concerted action of chromatin-modifying enzymes and noncoding RNAs. The novel exciting role of exosome-bound microRNA in mediating epigenetic information transfer is also discussed. Finally we show an overview of the epigenetic strategies and therapies that aim to potentiate muscle regeneration and counteract the progression of Duchenne Muscular Dystrophy (DMD).
\end{abstract}

\section{Introduction}

Epigenetic regulation of chromatin structure is fundamental to achieve the activation or repression of transcriptional programs governing cell development and differentiation. Changing cell phenotype without affecting genotype, epigenetics controls the spatial and temporal regulation of gene expression that ensures the quality, stability, and heritability of cell identity. At least three systems, including DNA methylation, posttranslational histone tail modifications, and noncoding RNA, are currently involved in epigenetic regulation [1]. Epigenetic changes occur naturally in normal development and health but can also be influenced by several factors including aging and diseases. Indeed aberrant epigenetic control can cause abnormal activation or silencing of genes. Importantly epigenetic modifications are reversible and sensitive to the environment, having therefore the potential to be therapeutically manipulated. Thus, epigenetics is currently a hot topic for research and the number of studies relating to various models of epigenetic regulation is tremendously increasing. Moreover, advances in genomewide technologies trying to elucidate epigenetic profiling (i.e., ChIP-seq, ChIA-PET, and Hi-C) hold the promise to deeply clarify the epigenetic control of cellular identity in health and disease.

Adult stem cells are candidate targets of epigenetic therapies toward repairing injured or diseased tissues, so they represent a key issue in regenerative medicine. In this context, skeletal muscle regeneration provides an insightful model for the study of the epigenetic events supporting the synchronized activation and repression of gene expression during stem cells differentiation. Indeed adult muscle stem cells remain in an embryonic-like state during development with the long-term ability to self-renewal and differentiate in response to injury [2]. A global genome reorganization allows activation, proliferation, and subsequent differentiation of quiescent progenitor muscle cells into functional multinucleated myofibers. Satellite cells are the main source of muscle stem cells (MuSCs) that regenerate adult skeletal muscles during postnatal life [3]. Intriguingly during aging or muscular disorders in which there is a chronic loss of skeletal muscle structure, the satellite cells function is compromised [4] even if their endogenous capacity to regenerate is not affected [5]. In fact it was demonstrated that the muscle 
environment is critical to permit effective muscle regeneration [6]. In particular the recently identified population of muscle interstitial cells, named fibroadipogenic progenitors (FAPs), plays a key role in supporting MuSCs activity and regeneration. However, in chronic muscle damage these cells lose their ability to support MuSCs mediated muscle regeneration and differentiate into fibroblasts and adipocytes [7-11]. An extensive analysis of the epigenome of these cells in healthy and diseased muscles is currently missing and would be crucial to better understand and pharmacologically manipulate changes that affect their regeneration activity.

The most severe neuromuscular disease is the Duchenne Muscular Dystrophy (DMD), a rare X-linked genetic disease caused by mutations in the dystrophin gene. DMD is characterized by a rapid progression of muscle degeneration that leads to the loss of ambulation and death within the second decade of life. In DMD the unbalanced regeneration of muscles exposed to continuous waves of degeneration leads to replacement of contractile myofibers with fibrotic and fatty tissue $[12,13]$. Nowadays there is not available cure for dystrophic patients and treatment is restricted to strategies that counteract the progression of the disease. The only therapy is limited to using corticosteroids as drugs to improve muscle strength. A huge number of studies for the treatment of the muscular disease are arising and some of them are undergoing clinical investigation. Gene and celltherapies, acting to repair the genetic defect, represent the most promising curative approach in the treatment of DMD but are still far from clinical translation [14].

Otherwise, pharmacological approaches that target the pathological consequences of the genetic defect are easy prompt to clinical practice translation. Actually, the pharmacological therapy for DMD includes nitric oxide (NO) administration, insulin-like growth factor 1 (IGF-1) stimulation, and myostatin inhibition in way to increase skeletal muscle mass; otherwise, therapies leading the inhibition of the transforming growth factor-beta (TGF $\beta$ ) pathway, modulation of nuclear factor $-\kappa \mathrm{B}(\mathrm{NF}-\kappa \mathrm{B})$, and tumor necrosis factor- $\alpha$ (TNF- $\alpha$ ) signalling are used to reduce fibrosis and inflammation in muscle [15].

However, the major limitation of manipulating target pathways consists in the lack of selectivity resulting in undesired side effects. Thus, regenerative medicine is providing novel strategies developing several epigenetic drugs aimed to manipulate the chromatin targets of individual signalling pathways. In the context of DMD, Histone Deacetylase Inhibitors (HDACi) are emerging as promising treatment to increase the functional and morphological recovery of dystrophic muscles [16-18]. Most of the beneficial effects of HDACi arise from their ability to activate a microRNASWI/SNF based epigenetic network in FAPs that redirects their lineage commitment from a fibroadipogenic toward a myogenic fate [19].

MicroRNAs (miRs) belong to the small noncoding RNAs family and are known to control numerous biological processes representing the most prevalent regulatory mechanism of mRNA availability in cells [20]. Apart from their role in regulating cell-autologous epigenetic events, miRs are involved also in cell-to-cell communication being involved in epigenetic regulation of recipient cells. miR shuttle between cells appears to be preserved and mediated by extracellular vesicles (i.e., exosomes) that are emerging as potent genetic transfer agents [21]. Interestingly stem cell-derived extracellular vesicles appear to be naturally equipped to mediate tissue regeneration and recent evidence suggests their therapeutic potential for targeted delivery of exogenous miRs [22].

In this review, we will focus on the principal epigenetic regulatory mechanisms underpinning skeletal muscle regeneration and their potential manipulation to develop pharmacological therapies for the treatment of DMD.

\section{Chromatin-Modifying Enzymes: Epigenetic Writers and Erasers Regulating Cell Epigenome}

The temporally regulated gene expression that controls pluripotency and differentiation is achieved by highly coordinated epigenetic events that ensure lineage commitment and cell fate determination. Epigenetic regulation of chromatin structure is fundamental to the activation or repression of specific transcriptional programs and is mainly controlled by chromatin-modifying enzymes that induce DNA methylation, posttranslational histone tail modifications, and nucleosome remodelling.

DNA methylation is a heritable, yet reversible, epigenetic modification that plays a central role in transcriptional repression. DNA methyltransferases (DNMTs) catalyze the transfer of a methyl group from cofactor $S$ adenosylmethionine to carbon 5 of the cytosines $(5 \mathrm{mC})$ that typically reside within a $\mathrm{CpG}$ dinucleotide. Regions of high CpG density, known as $\mathrm{CpG}$ islands, are typically devoid of DNA methylation [23]. Conversely, genes regulated by methylation usually contain low $\mathrm{CpG}$ density promoters and are demethylated and expressed in a cell-type-specific manner during differentiation [24-26]. This process is well illustrated during skeletal muscle cell fate commitment and differentiation. During development, pluripotent cells show a progressive loss of methylation leading to muscle stem cells with a unique DNA methylation signature associated with its specialized functions. Specific-myogenic factors such as MyoD and Myogenin are activated in a demethylationdependent manner driving the activation of the myogenic program (reviewed in [27]). Simultaneously, myogenesis is accompanied by DNA methylation of pluripotency and developmental genes (i.e., Hox genes) [28]. Seminal works demonstrated that treatment with 5-azacytidine, a potent inhibitor of DNA methylation, triggers myogenic differentiation in nonmuscle cells, linking for the first time $M y o D$ tissue-specific demethylation and cell fate commitment [2931]. DNA demethylation may also provide a transcriptionally poised state of muscle fiber genes that would be activated during differentiation, upon the acquisition of transcription factors and positive histone marks.

Indeed changes in DNA methylation and histone modifications strongly cooperate to achieve the global genome 
reorganization of progenitor cells necessary to establish myogenic identity, proliferation, and subsequent differentiation.

Satellite cells represent the main source of stem cells for adult muscle regeneration. Following muscle injury, they are readily activated and induced to proliferate and differentiate in multinucleated myofibers $[32,33]$. The myogenic lineage in satellite cells is determined by the expression of Pax 3 and Pax7 genes, while the expression of basic helix-loop-helix Myogenic Regulatory Factors (MRFs; MyoD, Myf5, Myogenin, and MRF4) in cooperation with myocyte enhancer factor-2 (MEF2) family proteins confers their ability to form differentiated myofibers [34]. Satellite cells activation is reflected in drastic changes at specific chromatin regions via the action of chromatin-modifying enzymes [35].

There are several classes of posttranslational histone modifications (i.e., phosphorylation, acetylation, methylation, and ubiquitylation) that affect chromatin structure and accessibility [36].

Histone acetylation has generally been linked to transcriptional active chromatin and is dynamically regulated by the opposing activities of histone acetyltransferases (HATs) and histone deacetylases (HDACs). A large amount of work illustrated the fundamental role of HATs and HDACs in regulating muscle development and differentiation. HATs catalyse the transfer of acetyl groups to lysine residues of histones, resulting in the relaxation of chromosomal DNA permissive for transcription. The histone acetyltransferases p300/CBP and PCAF activate muscle gene expression by acetylation of MyoD and modulation of its recruitment at target loci [37]. Interestingly recent studies have highlighted the ability of MyoD to preset the chromatin landscape of myoblasts for the activation of muscle-specific genes. Indeed genome-wide binding of MyoD has been associated with HATs recruitment and regional histone acetylation [38], while MyoD-bound distal enhancers have been linked to the recruitment of additional transcription factors and the regional enrichment of $\mathrm{H} 3 \mathrm{~K} 4$ monomethylation ( $\mathrm{H} 3 \mathrm{~K} 4 \mathrm{mel})$ and $\mathrm{H} 3 \mathrm{~K} 27$ acetylation (H3K27ac), two typical markers of active enhancers [39].

HDACs function to reverse histone acetylation, causing chromosomal DNA condensing and preventing the unscheduled transcriptional activation of muscle-specific genes in undifferentiated cells. There are currently 18 known human HDACs grouped into four classes [40]. Classes I, II, and IV HDACs are zinc-dependent proteins, while class III HDACs require $\mathrm{NAD}^{+}[41]$.

Interestingly, class I HDACs (HDAC1 and HDAC2) show constitutive nuclear localization and preferentially associate with MyoD [42], while class II HDAC members (HDAC4 and 5) shuttle between the nucleus and the cytoplasm and are dedicated repressors of MEF2-dependent transcription [43, 44]. Upon differentiation, displacement of HDACs from the chromatin of target genes correlates with the hyperacetylation at muscle loci and activation of muscle gene transcription (i.e., Myogenin and Myosin heavy chain) [45].

Given the importance of the balance between acetylation and deacetylation in regulating muscle gene transcription, HDACi are emerging as promising drugs to manipulate the regenerative potential of stem cells in diseased muscle [15] (see below). Despite the general assumption that HDAC inhibition would indiscriminately cause a global hyperacetylation in all organs and tissues, several studies revealed a surprising selective effect of HDACi on embryonic and adult stem cells [46] and in particular on genes with bivalence or with preexisting activator marks [15, 47]. Accordingly genome-wide Chip-seq analysis in myoblasts showed that the large majority of HDACi induced genes were involved in the myogenic differentiation program (i.e., Myosin 7 (MyH7), Enolase 3 (ENO3), and Myomesin 1 (MYOM1)) and showed bivalent (42\%) or active (57\%) epigenetic marks [48]. This data indicates that, in myoblasts, HDACi enforce and anticipate the expression of genes that are normally induced during differentiation. A bivalent chromatin structure builds an epigenetic signature that identifies genes poised for transcription typically enriched in stem cells. Poised genes show bivalent promoters marked by the presence of both active and repressive histone methylation marks $[49,50]$.

Methylation is linked to both active and inactive chromatin regions depending on the specific histone and lysine residue that is targeted. In particular, tri-methylation of lysine 4 on histone $\mathrm{H} 3$ ( $\mathrm{H} 3 \mathrm{~K} 4 \mathrm{me} 3)$ is associated with transcriptionally active promoters, while tri-methylation of lysine 27 (H3K27me3) leads to chromatin condensation [51].

Differentiated cells usually resolve bivalent promoters into an active or repressive state $[52,53]$ and become resistent to HDACi treatments. Indeed HDACi have been shown to potentiate myogenesis and the gene expression profile selectively in proliferating myoblasts and not in terminally differentiated myotubes. As differentiation precedes, muscle specific genes (i.e., Myogenin and MCK) gradually lose H3K27me3 and gain H3K4me3 [54]. Conversely, progenitorspecific transcription factors (i.e., Pax7) require H3K27me3mediated epigenetic repression for myotube maturation [55]. This process is finely regulated by the activity of two classes of histone lysine methyltransferases: the Polycomb group proteins responsible of $\mathrm{H} 3 \mathrm{~K} 27 \mathrm{me} 3$ for epigenetic silencing and the Trithorax group ( $\operatorname{Trx} G)$ which activates gene transcription catalyzing $\mathrm{H} 3 \mathrm{~K} 4 \mathrm{me} 3$.

Dynamic changes in the epigenetic landscape of muscle progenitors during differentiation are coordinated by extracellular signals that specifically target the activity and recruitment of chromatin modifier enzymes. For instance, the regeneration-activated p38 signalling targets multiple components of the myogenic transcriptosome that is assembled on the chromatin of muscle genes in response to locally released regeneration cues. $\mathrm{p} 38 \alpha / \beta$ kinases phosphorylate MEF2D mediating the recruitment of the Trithorax enzymatic subunit Ash2L to the chromatin of muscle genes [56]. Concomitantly p38 $\alpha$ kinase promotes the phosphorylation of EZH2, the enzymatic subunit of the Polycomb Repressive Complex 2 (PRC2), targeting Pax7 promoter for repression [55]. Finally p38 signalling promotes the recruitment of the chromatin remodelling SWI/SNF complex to the regulatory regions of MyoD-target muscle genes by the phosphorylation of BAF60c [57].

SWI/SNF complex comprises two mutually exclusive enzymatic sub-units (the ATPases Brgl and Brm) and several 


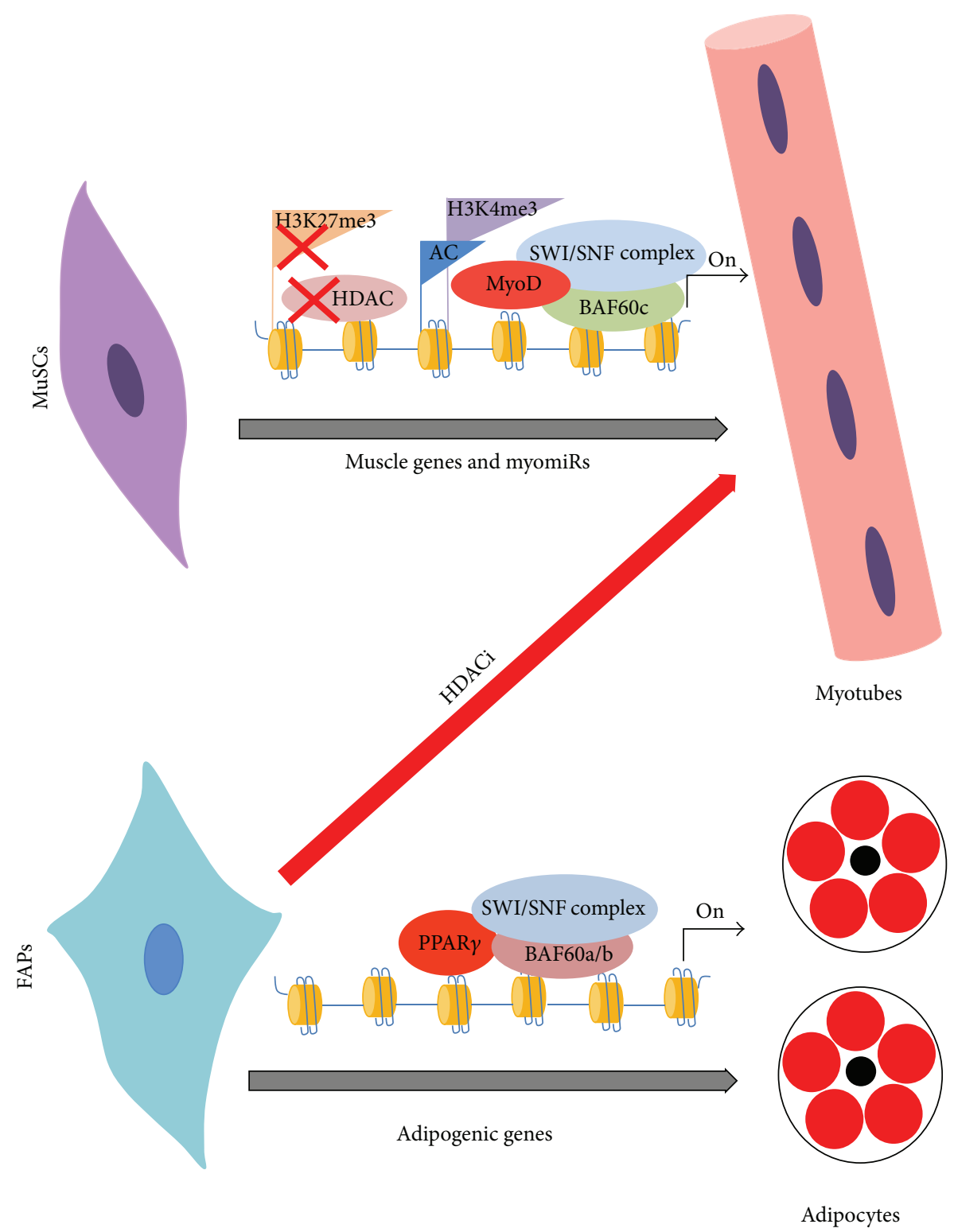

FIGURE 1: Epigenetic reprogramming of MuSCs and FAPs during differentiation. MuSCs adopt a chromatin permissive structure on muscle genes in which MyoD and BAF60c-based SWI/SNF complex promote transcription (on the top). FAPs differentiation into adipocytes is mediated by BAF60a/b-based SWI/SNF complex (on the bottom). HDACi treatment in dystrophic muscles activates a myomiR/MyoD/BAF60c network that, switching the BAF60 subunits assembled in the SWI/SNF complex, reprograms FAPs toward the acquisition of a myogenic phenotype.

Brg1/Brm associated factors (BAFs) [58]. In particular, three alternative variants of Baf60 sub-unit (BAF60a, BAF60b, and BAF60c) confer the affinity for tissue-specific transcription factors regulating lineage determination in many cell types $[19,59-61]$. BAF60c is essential to activate both skeletal and cardiac muscle programs $[57,61]$, while BAF60a and BAF60b activate alternative lineages, including lipid metabolism [62]. During embryo myogenesis, the negative regulation of BAF60a and BAF60b leads in progenitor cells the activation of a BAF60c-mediated muscle differentiation program [63].
Intriguingly our recent study demonstrated that BAF60 selection can drive lineage determination in a population of fibro-adipogenic progenitors (FAPs) resident in skeletal muscles. Favouring BAF60c incorporation in SWI/SNF complex at expense of $\mathrm{BAF} 60 \mathrm{a} / \mathrm{b}$ directs the switch from the fibroadipogenic to the myogenic lineage reducing fibrosis and fat deposition in dystrophic muscles (Figure 1) [19, 64]. These data suggest that therapeutic approaches aim to selectively target the combinatorial assembly of the SWI/SNF complex could be used to manipulate cell fate determination in several disorders. 


\section{Non-Coding RNAs as Epigenetic Regulators of Gene Expression}

A novel emerging level of gene expression regulation is mediated by non-coding RNAs (ncRNAs): functional RNA molecules not translated into proteins, composite of structural and regulatory RNAs. ncRNAs are divided by their size into long non-coding RNA (lncRNAs) greater than 200 nucleotides to over $100 \mathrm{~kb}$ in length and small non-coding $R N A$ (sncRNAs) with a non-coding transcript long less than 200 nucleotides [65].

LncRNAs localize both in the nucleus and cytoplasm and have roles in chromatin remodelling, transcription, intracellular trafficking and post-translational processes controlling cell identity and lineage commitment $[66,67]$. LncRNAs located in the nucleus regulate transcription recruiting chromatin-modifying enzymes or interacting with RNA sequences to influence their splicing. Many nuclear lncRNAs associate with EzH2/PRC2 and control the formation of nuclear compartments (i.e., speckles, para-speckles, polycomb bodies) [68, 69]. IncRNAs identified in cytoplasm regulate protein localization, mRNA translation and stability. Intriguingly it was recently described their role as sponge for miRNAs: reducing miRNAs levels, they inhibit the miRNAmRNA mediated target degradation [66]. IncRNAs strongly regulate $\mathrm{MuSCs}$ differentiation. The muscle-specific lincMD1 manages the time of muscle differentiation acting as a sponge to sequester miR-133 and miR-135 that regulate the expression of MAML1 and MEF2C, pro-myogenic transcription factors [66]. IncRNAs transcribed from MyoD enhancer (Enhancer RNAs (eRNAs)) regulate also MyoD and Myogenin expression [70].

sncRNAs family include transfer RNAs (tRNAs) and ribosomal RNAs (rRNAs), as well as microRNAs (miRNAs), Piwi-interacting RNAs (piRNAs), small interfering RNAs (siRNAs), small nuclear RNAs (snRNAs) [65]. Recent studies suggest the existence of thousands of ncRNAs, many of them involved in the epigenetic regulation of development, physiology, tissue regeneration and disease $[71,72]$. The most studied small non-coding RNAs are the miRNAs, molecules containing about 22 nucleotides, expressed in eukaryotes and found well conserved in plants and animals. miRNAs regulate numerous biological processes inhibiting translation of their target mRNA and also mediating their degradation through recognition of imperfect complementary sites, usually located in the $3^{\prime}$-untranslated regions $[73,74]$. It seems that miRNAs regulate the expression of more than $50 \%$ of mammalian genes making them the most prevalent regulatory mechanism of mRNA availability $[20,75,76]$.

miRNAs may fine tuning distinct processes targeting specific epigenetic regulators: DNA methylases, PRC components, Histone Deacetylases and chromatin remodelling complexes members [77]. Given their consistent epigenetic role, miRNAs are important regulators of embryonic and adult myogenesis [78] controlling MuSCs quiescence, proliferation and differentiation [79, 80]. For instance, Rando's group identified in a microarray expression study, about twenty quiescence-specific miRNAs that actively maintain the quiescent state of satellite cells (i.e., miR-489 that targets the oncogene Dek) and $351 \mathrm{miRNAs}$ regulating satellite cells activation [81].

One crucial step for MuSCs activation is miR-31 downregulation that allows Myf5 translation in myoblasts [82]. Myf5, together with MyoD, leads the activation of $\mathrm{miR}-133 \mathrm{a} / \mathrm{b}$ that inhibit the adipogenic regulator PRDM16 preventing muscle progenitors cells commitment to adipose cell fate [83, 84]. miR-133 controls also myoblasts proliferation acting as SRF regulator [85] During myogenesis, miR-1, miR-29 and miR206 target HDAC4 promoting the activity of the myogenic transcriptional elements Mef-2 and MRFs [86]. MRFs in turn regulate the expression of miR-1, miR-133a/b and miR206, muscle specific miRNA defined as "myomiRs". Finally miR-1 and miR-206 control Pax3/7 repression [87] while miR-26a targets the Ezh2 methyltransferase, to allow muscle differentiation $[54,88]$.

Different studies demonstrated that miRNAs could modulate the composition of SWI/SNF chromatin remodelling complexes in a way to epigenetically reprogram cell fate determination. Crabtree showed a microRNA mediated switching of chromatin-remodelling complexes in neural development: miR-9 and miR-124 target BAF53a sub-unit driving differentiation of progenitor cells into neurons [89]. Similarly our group identified in muscle interstitial FAPs an analogous miR-based mechanism that regulates the balance toward myogenic versus alternative fates (fibro-adipogenesis). In FAPs myomiRs (miR-1,2as, miR-133a and miR-206) favour the composition of the pro-myogenic BAF60c-SWI/SNF complex by targeting the alternative BAF60a and BAF60b variants [19]. Similarly in Embryonic muscle progenitors myomiRs negatively regulate $\mathrm{BAF} 60 \mathrm{a} / \mathrm{b}$ to promote the BAF60c-SWI/SNF complex [63].

Interestingly miRNAs derived from various tissues and organs, being stable and resistant to nuclease digestion, are easily detectable in both plasma and serum and may serve as diseases biomarker. Indeed circulating miRNAs profile dynamically change in many diseases such as cancer, myocardial infarction, heart failure, myotonic Distrophy type I and DMD [90-95]. MyomiRs for instance, have been identified in serum of muscular dystrophy animal models and patients where they are passively released as a consequence of myofibers degeneration and breakdown. Their putative active role is still unkown and currently they are proposed as novel diagnostic markers of disease progression. Indeed myomiRs detection in serum is inversely correlated to muscle health, representing a more sensible biomarker than the commonly used Creatine Kinase (CK) [94, 96].

\section{Extracellular Vesicles for Genetic Information Transfer and Cell Phenotype Modulation}

Extracellular vesicles are emerging as potent sources of genetic information transfer between cells and are involved in regulating stem cell plasticity via epigenetic reprogramming and their ability to alter gene regulatory networks [21]. Cellderived vesicles such as exosomes and microvesicles possess the capability to mediate intercellular communication by 
fusing with the plasma membrane of recipient cells and subsequently delivering their cargo, consisting of functional proteins, mRNAs and miRNAs able to modulate gene expression and cell phenotype [97]. Exosomes are homogenous small particles, usually 30 to $100 \mathrm{~nm}$ in size, of endosomal origin. Microvesicles, instead, constitute a larger and heterogeneous population of extracellular vesicles, 50 to $1000 \mathrm{~nm}$ in size, and are directly produced through the plasma membrane budding [22].

Multiple cell types have been described to release vesicles in extracellular medium, including mesenchymal cells, adipocytes, fibroblasts, immune cells and myoblasts. Little is known about vescicles regulation of MuSCs in health and diseased muscles. However several studies reporting muscleexosomes are emerging [98-100]. Myoblasts and myotubes use exosome clustered miRNAs as "endocrine signals" to control important signaling pathways (i.e., Wnt signaling pathway) for muscle homeostasis and regeneration. MiRNAs secreted in exosome by myotubes are functionally able to silence the HDAC Sirtl in myoblasts, controlling their commitment to differentiation [88]. Muscle behaviour is also influenced by vesicles released from different sources, like mesenchymal stem cells. Indeed it has been recently shown that miRNAs (i.e., miR-494 and myomiRs) released in exosomes from mesenchymal stem cells promote muscle regeneration following injury by enhancing myogenesis and angiogenesis [101]. Indeed exosomes appear to be naturally equipped to mediate tissue regeneration and their cargo constitute a rapid response, protected by the oxidative environment, to initiate tissue repair [102]. Vesicles from mesenchymal stem cells were found to confer therapeutic benefit in a range of different diseases: kidney [103-105] and hepatic injuries [106] myocardial ischaemia and infarction [107-109] and peripheral arterial disease [110]; this "regenerative" effect is mainly due to the ability of extracellularvesicles to induce phenotypic changes in local stem cells through epigenetic reprogramming to stimulate tissue repair and regeneration [111]. Notably, the transfer of tissue-specific mRNAs, miRNAs and protein-based transcription factors through the extracellular microvesicles was shown to induce phenotype change in bone marrow cells when co-cultured with cells derived from various tissues (brain, heart, liver and lung) [112-114].

Extracellular vesicles mediate communication even between distally located cells and tissue and can be found in many biological fluids including blood, saliva, urine, and breast milk [22]. For instance tumor cells can induce apoptosis in distal skeletal muscles via exosome assembled miR-21, which signals through the Toll-like 7 receptor (TLR7) on myoblasts to promote cell death and cancer cachexia [115].

Given their ability to be readily isolated from most body fluids, circulating miRNAs packed into exosomes are emerging as useful biomarkers to determine the development and progression of various diseases. Moreover, their natural role in transferring genetic material both locally and systemically has inspired pharmacological strategies to exploit these vesicles as therapeutic agents via the introduction of exogenous genetic cargoes such as siRNA (see below).

\section{Epigenetic Therapies and Future Perspectives for Muscle Regeneration in DMD}

In last years, regenerative medicine focused on the study of plasticity of stem cells epigenome and the recent findings lead the researchers to concentrate on strategies aimed to reprogram the stem cell fate in numerous diseases.

Myogenesis is coordinated by a complex interplay between epigenetic events that are crucial to control lineage determination and differentiation of adult stem cells. Basic research and recent studies of next generation sequencing are clarifying the fine epigenetic regulation of myogenesis and which are the epigenetic players that create changes in the epigenome opening new therapeutic options in muscle diseases as DMD.

HDACi are considered as the first generation of epigenetic drugs with proven clinical efficacy in the treatment of some lymphoid malignancies [116] and are now in clinical trials for a number of other diseases including DMD. Indeed preclinical studies in dystrophic mice (mdx) showed their ability to alleviate both morphological and functional consequences of the primary genetic defect $[16,17]$. The current availability of HDACi in clinical practice gave the opportunity for an immediate translation of these drugs into pharmacological treatments of DMD in human patients. The HDACi ITF2357 (Givinostat) represents the first epigenetic drug included into a study therapy for DMD.

Givinostat has already been tested in pediatric populations and received an Orphan Drug Designation by EMA for the treatment of systemic-onset juvenile idiopathic arthritis (SOJIA) [117, 118]. This knowledge encouraged its traslation into a phase I/II clinical trial with children affected by DMD (ClinicalTrials.gov identifier: NCT01761292). After one year of treatment Givinostat efficacy has been monitored showing very promising results on muscle histology and functionality without severe adverse effects on children health; thus the trial has been prolonged for a second year. Obviously this study requires defining the activity of Givinostat in long-term treatments to assess its persistent effect in dystrophic muscles and to monitor possible adverse events.

The functional characterization of the recently identified epigenetic network that determines the ability of HDACi to promote regeneration of dystrophic muscles, at expense of fibrosis and fat deposition, highlights the role of FAPs as key cellular mediators of HDACi activity and DMD progression $[18,19]$.

FAPs are multipotent mesenchymal cells located in muscle interstitium with the ability to proliferate and support satellite cells mediated muscle regeneration in response to local injury or disease. However, beyond their beneficial role, FAPs have been shown to be the major source of fibroadipocytes in degenerating muscles $[9,10]$.

Importantly we have demonstrated that treatment with HDACi at early stages of DMD induces in FAPs a myogenic fate at expense of their fibro-adipogenic lineage. HDACi de-repress a latent myogenic program by activating a MyoD/BAF60c/myomiR network that leads muscle 


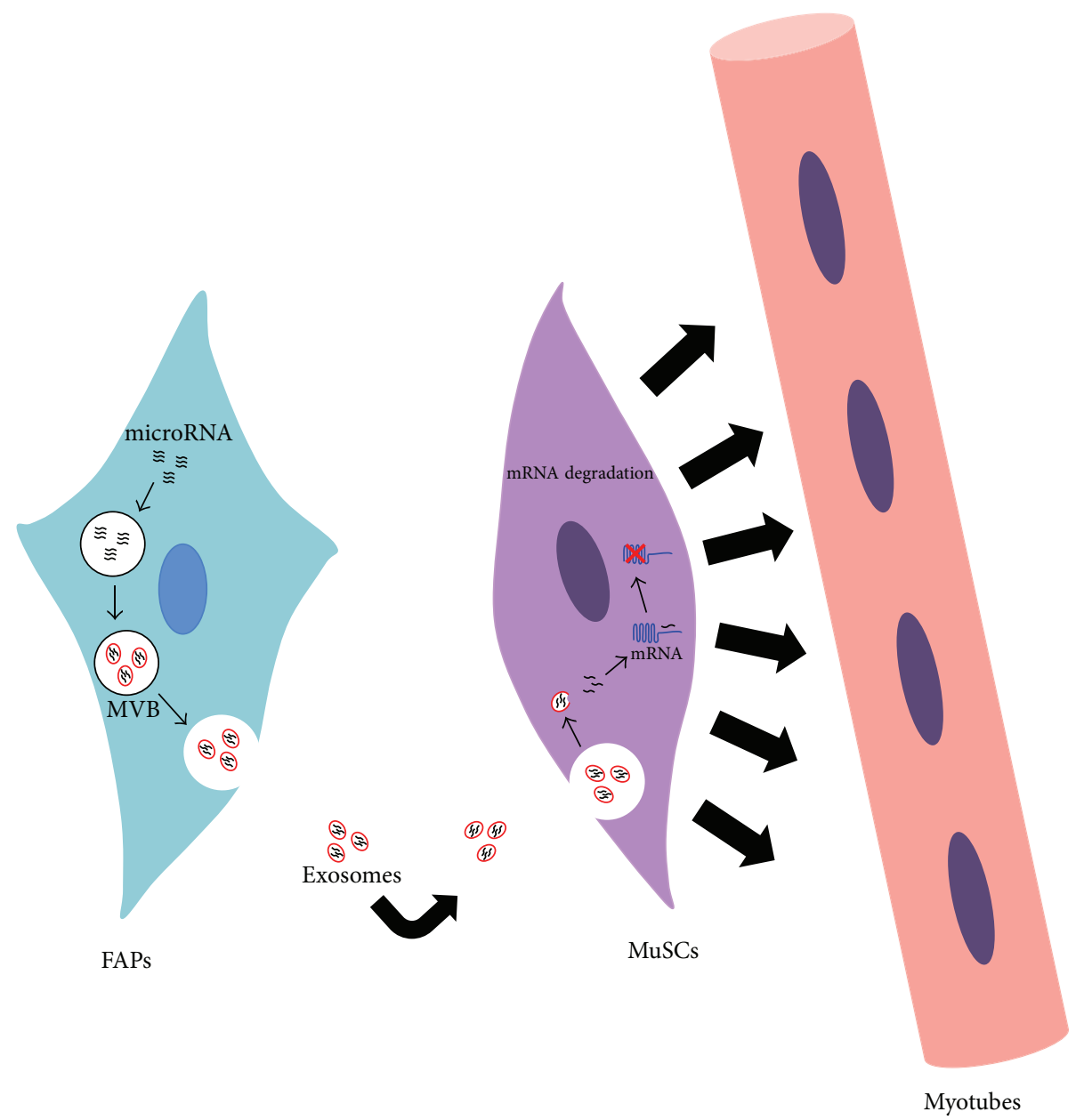

FIGURE 2: Exosomes as putative mediators of the functional interaction between FAPs and MuSCs. In this model, exosomes released by activated FAPs support myoblasts differentiation through a mechanism by which their cargo of miRNA can be transferred to MuSCs.

differentiation. Indeed HDACi induce MyoD and BAF60c expression, two core components of the myogenic transcriptional machinery, and up-regulate myomiRs (miR-1.2, -133 and -206), which target the alternative BAF60 variants A and B. Switching of the BAF60 sub-units assembled in the SWI/SNF complex reprograms FAPs toward the acquisition of a pro-myogenic phenotype. However the progressive impairment of the integrity of this network prevents HDACi efficacy at late stages of DMD. Indeed, with the progress of the disease FAPs becomes resistant to HDACi and acquire a constitutive fibro-adipogenic lineage replacing the muscle loss with fatty and fibrotic tissues [18]. Importantly, transplantation of "young" FAPs into muscles of "old" dystrophic mice, restored the ability of HDACi to promote regeneration at advanced stages of disease [18]. This suggests that a powerful future therapeutic strategy will be to epigenetically reprogram aged FAPs with selective delivery of Baf60c and myomRs. In this context the natural ability of exosomes to transfer material both locally and systemically encourage the possibility of exploiting these vesicles for therapeutic purposes.
While these data provide new insight into the molecular pathogenesis of DMD and therapeutic approaches to delay the disease, they also highlight the potential of miRs detection as clinical biomarkers of disease progression. The increase of circulating myomiRs in the peripheral blood of dystrophic patients correlates with the severity of the disease, suggesting that myomiR quantification in blood of DMD patients might represent a sensible diagnostic and prognostic marker [64, 94]. On the other hand our recent data, show a great increase of FAPs derived myomiRs in muscle interstitium of $\mathrm{mdx}$ mice after HDACi exposure suggesting an inverse correlation between local and circulating myomiRs [19]. This suggests that detection of muscular (local) versus circulating myomiRs could provide a novel more accurate biomarker for diagnosis of DMD progression and efficacy of therapeutic drugs [64].

MiR stability in extracellular environment seems to be preserved by vesicles budding and intriguingly myomiRs have been detected in vitro in exosomes released by mesenchymal cells to support myoblasts differentiation [101]. An analogous mechanism is probably involved in vivo between FAPs and MuSCs to promote muscle regeneration (Figure 2). 
It would be important to investigate if this functional crosstalk mediated by exosome is somehow affected in muscle disorders. Furthermore these data strongly encourage the possibility to re-engineer naturally derived exosomes for DMD epigenetic therapy.

\section{Conclusions}

In the last years, great advances have been made in the comprehension of the epigenetic mechanisms regulating, via chromatin organization, different transcriptional programs. The functional characterization of the variety of epigenetic regulations in healthy and disease states has the prospect to identify novel targets for epigenetic-based therapies.

HDACi represent the first generation of epigenetic drugs. Their clinical efficacy is currently being tested in a phase I/II clinical trial on children affected by DMD. The proregenerative effects of HDACi are mediated by FAPs, a population of muscle-resident stem cells. However, dystrophic muscles at late stages of the disease are resistant to HDACiinduced beneficial effects. This unresponsiveness might be due to a decreased chromatin plasticity of FAPs caused by epigenetic silencing pathways. The identification of the epigenetic players preventing HDACi responsiveness at advanced stages of DMD will be crucial to devise new personalized and selective strategies to re-establish HDACi sensitivity. In this context, a comprehensive epigenetic mapping of the chromatin landscape of key populations involved in muscle regeneration is becoming urgent to identify in the near future both therapeutic effectiveness and inclusion criteria of DMD patients to epigenetic therapy.

Exosome-bound miRNAs are emerging as a crucial mechanism to transfer epigenetic information between cells. New evidence showing the therapeutic relevance of these vesicles in both unmodified and modified forms make them attractive therapeutic agents for further study. Moreover detection of specific miRNAs secreted in muscle interstitium and blood of dystrophic patients holds the promise to develop new painless methodologies, less invasive than classic biopsy, such as blood sampling or fine needle aspiration techniques, to diagnose DMD.

\section{Disclosure}

The authors declare that there is no conflict of interests regarding the publication of this paper.

\section{Conflict of Interests}

The authors declare that there is no conflict of interests regarding the publication of this paper.

\section{Acknowledgments}

This review has been supported by the AFM-Telethon Trampoline grant to Valentina Saccone. Valentina Saccone was supported by DPP NL Fellowship.

\section{References}

[1] M. J. Boland, K. L. Nazor, and J. F. Loring, "Epigenetic regulation of pluripotency and differentiation," Circulation Research, vol. 115, no. 2, pp. 311-324, 2014.

[2] S. B. P. Chargé and M. A. Rudnicki, "Cellular and molecular regulation of muscle regeneration," Physiological Reviews, vol. 84, no. 1, pp. 209-238, 2004.

[3] F. Le Grand and M. A. Rudnicki, "Skeletal muscle satellite cells and adult myogenesis," Current Opinion in Cell Biology, vol. 19, no. 6, pp. 628-633, 2007.

[4] A. E. H. Emery, “The muscular dystrophies," The Lancet, vol. 359, no. 9307, pp. 687-695, 2002.

[5] J. E. Morgan and P. S. Zammit, "Direct effects of the pathogenic mutation on satellite cell function in muscular dystrophy," Experimental Cell Research, vol. 316, no. 18, pp. 3100-3108, 2010.

[6] L. Boldrin, P. S. Zammit, and J. E. Morgan, "Satellite cells from dystrophic muscle retain regenerative capacity," Stem Cell Research, vol. 14, no. 1, pp. 20-29, 2015.

[7] B. Péault, M. Rudnicki, Y. Torrente et al., "Stem and progenitor cells in skeletal muscle development, maintenance, and therapy," Molecular Therapy, vol. 15, no. 5, pp. 867-877, 2007.

[8] K. J. Mitchell, A. Pannérec, B. Cadot et al., "Identification and characterization of a non-satellite cell muscle resident progenitor during postnatal development," Nature Cell Biology, vol. 12, no. 3, pp. 257-266, 2010.

[9] A. Uezumi, S.-I. Fukada, N. Yamamoto, S. Takeda, and K. Tsuchida, "Mesenchymal progenitors distinct from satellite cells contribute to ectopic fat cell formation in skeletal muscle," Nature Cell Biology, vol. 12, no. 2, pp. 143-152, 2010.

[10] A. W. B. Joe, L. Yi, A. Natarajan et al., "Muscle injury activates resident fibro/adipogenic progenitors that facilitate myogenesis," Nature Cell Biology, vol. 12, no. 2, pp. 153-163, 2010.

[11] M. S. Rodeheffer, “Tipping the scale: muscle versus fat," Nature Cell Biology, vol. 12, no. 2, pp. 102-104, 2010.

[12] I. Dalkilic and L. M. Kunkel, "Muscular dystrophies: genes to pathogenesis," Current Opinion in Genetics and Development, vol. 13, no. 3, pp. 231-238, 2003.

[13] E. P. Hoffman, R. H. Brown Jr., and L. M. Kunkel, "Dystrophin: the protein product of the duchenne muscular dystrophy locus," Cell, vol. 51, no. 6, pp. 919-928, 1987.

[14] S. Benedetti, H. Hoshiya, and F. S. Tedesco, "Repair or replace? Exploiting novel gene and cell therapy strategies for muscular dystrophies," The FEBS Journal, vol. 280, no. 17, pp. 4263-4280, 2013.

[15] S. Consalvi, V. Saccone, and C. Mozzetta, "Histone deacetylase inhibitors: a potential epigenetic treatment for Duchenne muscular dystrophy," Epigenomics, vol. 6, no. 5, pp. 547-560, 2014.

[16] S. Consalvi, C. Mozzetta, P. Bettica et al., "Preclinical studies in the mdx mouse model of duchenne muscular dystrophy with the histone deacetylase inhibitor givinostat," Molecular Medicine, vol. 19, no. 1, pp. 79-87, 2013.

[17] G. C. Minetti, C. Colussi, R. Adami et al., "Functional and morphological recovery of dystrophic muscles in mice treated with deacetylase inhibitors," Nature Medicine, vol. 12, no. 10, pp. 1147-1150, 2006.

[18] C. Mozzetta, S. Consalvi, V. Saccone et al., "Fibroadipogenic progenitors mediate the ability of HDAC inhibitors to promote regeneration in dystrophic muscles of young, but not old $\mathrm{Mdx}$ mice," EMBO Molecular Medicine, vol. 5, no. 4, pp. 626-639, 2013. 
[19] V. Saccone, S. Consalvi, L. Giordani et al., "HDAC-regulated myomiRs control BAF60 variant exchange and direct the functional phenotype of fibro-adipogenic progenitors in dystrophic muscles," Genes and Development, vol. 28, no. 8, pp. 841-857, 2014.

[20] V. Ambros, "microRNAs: tiny regulators with great potential," Cell, vol. 107, no. 7, pp. 823-826, 2001.

[21] Y. Lee, S. El Andaloussi, and M. J. A. Wood, "Exosomes and microvesicles: extracellular vesicles for genetic information transfer and gene therapy," Human Molecular Genetics, vol. 21, no. 1, Article ID dds317, pp. R125-R134, 2012.

[22] A. V. Vlassov, S. Magdaleno, R. Setterquist, and R. Conrad, "Exosomes: current knowledge of their composition, biological functions, and diagnostic and therapeutic potentials," Biochimica et Biophysica Acta, vol. 1820, no. 7, pp. 940-948, 2012.

[23] B. E. Bernstein, T. S. Mikkelsen, X. Xie et al., "A bivalent chromatin structure marks key developmental genes in embryonic stem cells," Cell, vol. 125, no. 2, pp. 315-326, 2006.

[24] S. D. Fouse, Y. Shen, M. Pellegrini et al., "Promoter CpG methylation contributes to ES cell gene regulation in parallel with Oct4/Nanog, PcG complex, and histone H3 K4/ K27 trimethylation," Cell Stem Cell, vol. 2, no. 2, pp. 160-169, 2008.

[25] T. S. Mikkelsen, M. Ku, D. B. Jaffe et al., "Genome-wide maps of chromatin state in pluripotent and lineage-committed cells," Nature, vol. 448, no. 7153, pp. 553-560, 2007.

[26] K. L. Nazor, G. Altun, C. Lynch et al., "Recurrent variations in DNA methylation in human pluripotent stem cells and their differentiated derivatives," Cell Stem Cell, vol. 10, no. 5, pp. 620634, 2012.

[27] E. Carrió and M. Suelves, "DNA methylation dynamics in muscle development and disease," Frontiers in Aging Neuroscience, vol. 7, article 19, 2015.

[28] K. Tsumagari, C. Baribault, J. Terragni et al., "DNA methylation and differentiation: HOX genes in muscle cells," Epigenetics and Chromatin, vol. 6, article 25, 2013.

[29] C.-P. Chiu and H. M. Blau, "5-azacytidine permits gene activation in a previously non-inducible cell type," Cell, vol. 40, no. 2, pp. 417-424, 1985.

[30] P. G. Constantinides, P. A. Jones, and W. Gevers, "Functional striated muscle cells from non myoblast precursors following 5 azacytidine treatment," Nature, vol. 267, no. 5609, pp. 364-366, 1977.

[31] B. P. Brunk, D. J. Goldhamer, and C. P. Emerson Jr., "Regulated demethylation of the myoD distal enhancer during skeletal myogenesis," Developmental Biology, vol. 177, no. 2, pp. 490-503, 1996.

[32] A. Mauro, "Satellite cell of skeletal muscle fibers," The Journal of Biophysical and Biochemical Cytology, vol. 9, no. 2, pp. 493-495, 1961.

[33] M. H. Snow, "An autoradiographic study of satellite cell differentiation into regenerating myotubes following transplantation of muscles in young rats," Cell and Tissue Research, vol. 186, no. 3, pp. 535-540, 1978.

[34] A. S. Brack and T. A. Rando, "Intrinsic changes and extrinsic influences of myogenic stem cell function during aging," Stem Cell Reviews, vol. 3, no. 3, pp. 226-237, 2007.

[35] V. Sartorelli and A. H. Juan, "Sculpting chromatin beyond the double helix: epigenetic control of skeletal myogenesis," Current Topics in Developmental Biology, vol. 96, pp. 57-83, 2011.

[36] T. Kouzarides, "Chromatin modifications and their function," Cell, vol. 128, no. 4, pp. 693-705, 2007.
[37] T. A. McKinsey, C. L. Zhang, and E. N. Olson, "Control of muscle development by dueling HATs and HDACs," Current Opinion in Genetics and Development, vol. 11, no. 5, pp. 497-504, 2001.

[38] Y. Cao, Z. Yao, D. Sarkar et al., "Genome-wide MyoD binding in skeletal muscle cells: a potential for broad cellular reprogramming," Developmental Cell, vol. 18, no. 4, pp. 662-674, 2010.

[39] R. Blum, V. Vethantham, C. Bowman, M. Rudnicki, and B. D. Dynlacht, "Genome-wide identification of enhancers in skeletal muscle: the role of MyoD1," Genes and Development, vol. 26, no. 24, pp. 2763-2779, 2012.

[40] W. S. Xu, R. B. Parmigiani, and P. A. Marks, "Histone deacetylase inhibitors: molecular mechanisms of action," Oncogene, vol. 26, no. 37, pp. 5541-5552, 2007.

[41] J. S. Carew, F. J. Giles, and S. T. Nawrocki, "Histone deacetylase inhibitors: mechanisms of cell death and promise in combination cancer therapy," Cancer Letters, vol. 269, no. 1, pp. 7-17, 2008.

[42] A. Mal, M. Sturniolo, R. L. Schiltz, M. K. Ghosh, and M. L. Harter, "A role for histone deacetylase HDAC1 in modulating the transcriptional activity of MyoD: inhibition of the myogenic program," The EMBO Journal, vol. 20, no. 7, pp. 1739-1753, 2001.

[43] T. A. McKinsey, C. L. Zhang, and E. N. Olson, "Activation of the myocyte enhancer factor-2 transcription factor by calcium/calmodulin-dependent protein kinase-stimulated binding of 14-3-3 to histone deacetylase 5," Proceedings of the National Academy of Sciences of the United States of America, vol. 97, no. 26, pp. 14400-14405, 2000.

[44] J. Lu, T. A. McKinsey, C.-L. Zhang, and E. N. Olson, "Regulation of skeletal myogenesis by association of the MEF2 transcription factor with class II histone deacetylases," Molecular Cell, vol. 6, no. 2, pp. 233-244, 2000.

[45] T. A. McKinsey, C. L. Zhang, and E. N. Olson, "Signaling chromatin to make muscle," Current Opinion in Cell Biology, vol. 14, no. 6, pp. 763-772, 2002.

[46] G. Elaut, V. Rogiers, and T. Vanhaecke, "The pharmaceutical potential of histone deacetylase inhibitors," Current Pharmaceutical Design, vol. 13, no. 25, pp. 2584-2620, 2007.

[47] E. Karantzali, H. Schulz, O. Hummel, N. Hubner, A. K. Hatzopoulos, and A. Kretsovali, "Histone deacetylase inhibition accelerates the early events of stem cell differentiation: transcriptomic and epigenetic analysis," Genome Biology, vol. 9, no. 4, article R65, 2008.

[48] S. Consalvi, V. Saccone, L. Giordani, G. Minetti, C. Mozzetta, and P. L. Puri, "Histone deacetylase inhibitors in the treatment of muscular dystrophies: epigenetic drugs for genetic diseases," Molecular Medicine, vol. 17, no. 5-6, pp. 457-465, 2011.

[49] B. E. Bernstein, T. S. Mikkelsen, X. Xie et al., "A bivalent chromatin structure marks key developmental genes in embryonic stem cells," Cell, vol. 125, no. 2, pp. 315-326, 2006.

[50] V. Azuara, P. Perry, S. Sauer et al., "Chromatin signatures of pluripotent cell lines," Nature Cell Biology, vol. 8, no. 5, pp. 532$538,2006$.

[51] E. L. Greer and Y. Shi, "Histone methylation: a dynamic mark in health, disease and inheritance," Nature Reviews Genetics, vol. 13, no. 5, pp. 343-357, 2012.

[52] M. G. Lee, C. Wynder, D. A. Bochar, M.-A. Hakimi, N. Cooch, and R. Shiekhattar, "Functional interplay between histone demethylase and deacetylase enzymes," Molecular and Cellular Biology, vol. 26, no. 17, pp. 6395-6402, 2006. 
[53] F. Mohn, M. Weber, M. Rebhan et al., "Lineage-specific polycomb targets and de novo DNA methylation define restriction and potential of neuronal progenitors," Molecular Cell, vol. 30, no. 6, pp. 755-766, 2008.

[54] G. Caretti, M. Di Padova, B. Micales, G. E. Lyons, and V. Sartorelli, "The Polycomb Ezh2 methyltransferase regulates muscle gene expression and skeletal muscle differentiation," Genes and Development, vol. 18, no. 21, pp. 2627-2638, 2004.

[55] D. Palacios, C. Mozzetta, S. Consalvi et al., "TNF/p38 $\alpha$ / polycomb signaling to Pax7 locus in satellite cells links inflammation to the epigenetic control of muscle regeneration," Cell Stem Cell, vol. 7, no. 4, pp. 455-469, 2010.

[56] S. Rampalli, L. Li, E. Mak et al., "p38 MAPK signaling regulates recruitment of Ash2L-containing methyltransferase complexes to specific genes during differentiation," Nature Structural and Molecular Biology, vol. 14, no. 12, pp. 1150-1156, 2007.

[57] S. V. Forcales, S. Albini, L. Giordani et al., "Signal-dependent incorporation of MyoD-BAF60c into Brg1-based SWI/SNF chromatin-remodelling complex," The EMBO Journal, vol. 31, no. 2, pp. 301-316, 2012.

[58] W. Wang, Y. Xue, S. Zhou, A. Kuo, B. R. Cairns, and G. R. Crabtree, "Diversity and specialization of mammalian SWI/SNF complexes," Genes and Development, vol. 10, no. 17, pp. 21172130, 1996.

[59] P. L. Puri and M. Mercola, "BAF60 A, B, and Cs of muscle determination and renewal," Genes and Development, vol. 26, no. 24, pp. 2673-2683, 2012.

[60] A. S. Yoo, A. X. Sun, L. Li et al., "MicroRNA-mediated conversion of human fibroblasts to neurons," Nature, vol. 476, no. 7359, pp. 228-231, 2011.

[61] H. Lickert, J. K. Takeuchi, I. von Both et al., "Baf60c is essential for function of BAF chromatin remodelling complexes in heart development," Nature, vol. 432, no. 7013, pp. 107-112, 2004.

[62] S. Li, C. Liu, N. Li et al., "Genome-wide coactivation analysis of PGC- $1 \alpha$ identifies BAF60a as a regulator of hepatic lipid metabolism," Cell Metabolism, vol. 8, no. 2, pp. 105-117, 2008.

[63] K. Goljanek-Whysall, G. F. Mok, A. F. Alrefaei, N. Kennerley, G. N. Wheeler, and A. Münsterberg, "myomiR-dependent switching of BAF60 variant incorporation into Brgl chromatin remodeling complexes during embryo myogenesis," Development, vol. 141, no. 17, pp. 3378-3387, 2014.

[64] L. Giordani, M. Sandoná, A. Rotini, P. L. Puri, S. Consalvi, and V. Saccone, "Muscle-specific microRNAs as biomarkers of Duchenne Muscular Dystrophy progression and response to therapies," Rare Diseases, vol. 2, no. 1, Article ID e974969, 2014.

[65] J. Cao, "The functional role of long non-coding RNAs and epigenetics," Biological Procedures Online, vol. 16, article 11, 2014.

[66] M. Cesana, D. Cacchiarelli, I. Legnini et al., "A long noncoding RNA controls muscle differentiation by functioning as a competing endogenous RNA," Cell, vol. 147, no. 2, pp. 358-369, 2011.

[67] Y. Wang, Z. Xu, J. Jiang et al., "Endogenous miRNA sponge lincRNA-RoR regulates Oct4, Nanog, and Sox2 in human embryonic stem cell self-renewal," Developmental Cell, vol. 25, no. 1, pp. 69-80, 2013.

[68] J. Zhao, T. K. Ohsumi, J. T. Kung et al., "Genome-wide identification of polycomb-associated RNAs by RIP-seq," Molecular Cell, vol. 40, no. 6, pp. 939-953, 2010.

[69] V. Tripathi, J. D. Ellis, Z. Shen et al., "The nuclear-retained noncoding RNA MALAT1 regulates alternative splicing by modulating SR splicing factor phosphorylation," Molecular Cell, vol. 39, no. 6, pp. 925-938, 2010.
[70] K. Mousavi, H. Zare, S. Dell'orso et al., "eRNAs promote transcription by establishing chromatin accessibility at defined genomic loci," Molecular Cell, vol. 51, no. 5, pp. 606-617, 2013.

[71] M. Esteller, "Non-coding RNAs in human disease," Nature Reviews Genetics, vol. 12, no. 12, pp. 861-874, 2011.

[72] G. Sohi and F. J. Dilworth, "Noncoding RNAs as epigenetic mediators of skeletal muscle regeneration," FEBS Journal, vol. 282, no. 9, pp. 1630-1646, 2015.

[73] M. Selbach, B. Schwanhäusser, N. Thierfelder, Z. Fang, R. Khanin, and N. Rajewsky, "Widespread changes in protein synthesis induced by microRNAs," Nature, vol. 455, no. 7209, pp. 58-63, 2008.

[74] D. Baek, J. Villén, C. Shin, F. D. Camargo, S. P. Gygi, and D. P. Bartel, "The impact of microRNAs on protein output," Nature, vol. 455, no. 7209, pp. 64-71, 2008.

[75] A. E. Pasquinelli and G. Ruvkun, "Control of developmental timing by microRNAs and their targets," Annual Review of Cell and Developmental Biology, vol. 18, pp. 495-513, 2002.

[76] D. P. Bartel, "MicroRNAss: genomics,biogenesis, mechanism, and function," Cell, vol. 116, no. 2, pp. 281-297, 2004.

[77] F. Sato, S. S. Tsuchiya, S. J. Meltzer, and K. Shimizu, "microRNAs and epigenetics," The FEBS Journal, vol. 278, no. 10, pp. 15981609, 2011.

[78] G. Stefani and F. J. Slack, "Small non-coding RNAs in animal development," Nature Reviews Molecular Cell Biology, vol. 9, no. 3, pp. 219-230, 2008.

[79] S. Greco, M. De Simone, C. Colussi et al., "Common microRNA signature in skeletal muscle damage and regeneration induced by Duchenne muscular dystrophy and acute ischemia," The FASEB Journal, vol. 23, no. 10, pp. 3335-3346, 2009.

[80] I. Eisenberg, M. S. Alexander, and L. M. Kunkel, "miRNAS in normal and diseased skeletal muscle," Journal of Cellular and Molecular Medicine, vol. 13, no. 1, pp. 2-11, 2009.

[81] T. H. Cheung, N. L. Quach, G. W. Charville et al., "Maintenance of muscle stem-cell quiescence by microRNA-489," Nature, vol. 482, no. 7386, pp. 524-528, 2012.

[82] P. Daubas, C. G. Crist, L. Bajard et al., "The regulatory mechanisms that underlie inappropriate transcription of the myogenic determination gene Myf5 in the central nervous system," Developmental Biology, vol. 327, no. 1, pp. 71-82, 2009.

[83] H. Yin, A. Pasut, V. D. Soleimani et al., "MicroRNA-133 controls brown adipose determination in skeletal muscle satellite cells by targeting Prdm16," Cell Metabolism, vol. 17, no. 2, pp. 210-224, 2013.

[84] M. Trajkovski, K. Ahmed, C. C. Esau, and M. Stoffel, "MyomiR133 regulates brown fat differentiation through Prdm16," Nature Cell Biology, vol. 14, no. 12, pp. 1330-1335, 2012.

[85] J.-F. Chen, E. M. Mandel, J. M. Thomson et al., "The role of microRNA-1 and microRNA-133 in skeletal muscle proliferation and differentiation," Nature Genetics, vol. 38, no. 2, pp. 228-233, 2006.

[86] C. E. Winbanks, B. Wang, C. Beyer et al., “TGF- $\beta$ regulates miR206 and miR-29 to control myogenic differentiation through regulation of $\mathrm{HDAC}_{4}$," Journal of Biological Chemistry, vol. 286, no. 16, pp. 13805-13814, 2011.

[87] J.-F. Chen, Y. Tao, J. Li et al., "microRNA-1 and microRNA206 regulate skeletal muscle satellite cell proliferation and differentiation by repressing Pax7," Journal of Cell Biology, vol. 190, no. 5, pp. 867-879, 2010.

[88] F. W. Chung and R. L. Tellam, "microRNA-26a targets the histone methyltransferase enhancer of zeste homolog 2 during 
myogenesis," The Journal of Biological Chemistry, vol. 283, no. 15, pp. 9836-9843, 2008.

[89] A. S. Yoo, B. T. Staahl, L. Chen, and G. R. Crabtree, "MicroRNAmediated switching of chromatin-remodelling complexes in neural development," Nature, vol. 460, no. 7255, pp. 642-646, 2009.

[90] A. Perfetti, S. Greco, E. Bugiardini et al., "Plasma microRNAs as biomarkers for myotonic dystrophy type 1," Neuromuscular Disorders, vol. 24, no. 6, pp. 509-515, 2014.

[91] M. I. K. Anindo and A. Yaqinuddin, "Insights into the potential use of microRNAs as biomarker in cancer," International Journal of Surgery, vol. 10, no. 9, pp. 443-449, 2012.

[92] V. Di Stefano, G. Zaccagnini, M. C. Capogrossi, and F. Martelli, "MicroRNAs as peripheral blood biomarkers of cardiovascular disease," Vascular Pharmacology, vol. 55, no. 4, pp. 111-118, 2011.

[93] Y. D’Alessandra, P. Devanna, F. Limana et al., "Circulating microRNAs are new and sensitive biomarkers of myocardial infarction," European Heart Journal, vol. 31, no. 22, pp. 27652773, 2010.

[94] D. Cacchiarelli, I. Legnini, J. Martone et al., "miRNAs as serum biomarkers for Duchenne muscular dystrophy," $E M B O$ Molecular Medicine, vol. 3, no. 5, pp. 258-265, 2011.

[95] N. Vignier, F. Amor, P. Fogel et al., "Distinctive serum miRNA profile in mouse models of striated muscular pathologies," PLOS ONE, vol. 8, no. 2, Article ID e55281, 2013.

[96] H. Mizuno, A. Nakamura, Y. Aoki et al., "Identification of muscle-specific microRNAs in serum of muscular dystrophy animal models: promising novel blood-based markers for muscular dystrophy," PLoS ONE, vol. 6, no. 3, Article ID e18388, 2011.

[97] H. Valadi, K. Ekström, A. Bossios, M. Sjöstrand, J. J. Lee, and J. O. Lötvall, "Exosome-mediated transfer of mRNAs and microRNAs is a novel mechanism of genetic exchange between cells," Nature Cell Biology, vol. 9, no. 6, pp. 654-659, 2007.

[98] M. Guescini, D. Guidolin, L. Vallorani et al., "C2C12 myoblasts release micro-vesicles containing mtDNA and proteins involved in signal transduction," Experimental Cell Research, vol. 316, no. 12, pp. 1977-1984, 2010.

[99] D. P. Romancino, G. Paterniti, Y. Campos et al., "Identification and characterization of the nano-sized vesicles released by muscle cells," FEBS Letters, vol. 587, no. 9, pp. 1379-1384, 2013.

[100] A. Forterre, A. Jalabert, K. Chikh et al., "Myotube-derived exosomal miRNAs downregulate Sirtuin1 in myoblasts during muscle cell differentiation," Cell Cycle, vol. 13, no. 1, pp. 78-89, 2014.

[101] Y. Nakamura, S. Miyaki, H. Ishitobi et al., "Mesenchymal-stemcell-derived exosomes accelerate skeletal muscle regeneration," FEBS Letters, vol. 589, no. 11, pp. 1257-1265, 2015.

[102] F. T. Borges, S. A. Melo, B. C. Özdemir et al., "TGF- $\beta 1-$ Containing exosomes from injured epithelial cells activate fibroblasts to initiate tissue regenerative responses and fibrosis," Journal of the American Society of Nephrology, vol. 24, no. 3, pp. 385-392, 2013.

[103] S. Bruno, C. Grange, M. C. Deregibus et al., "Mesenchymal stem cell-derived microvesicles protect against acute tubular injury," Journal of the American Society of Nephrology, vol. 20, no. 5, pp. 1053-1067, 2009.

[104] S. Bruno, C. Grange, F. Collino et al., "Microvesicles derived from mesenchymal stem cells enhance survival in a lethal model of acute kidney injury," PLoS ONE, vol. 7, no. 3, Article ID e33115, 2012.
[105] S. Gatti, S. Bruno, M. C. Deregibus et al., "Microvesicles derived from human adult mesenchymal stem cells protect against ischaemia-reperfusion-induced acute and chronic kidney injury," Nephrology Dialysis Transplantation, vol. 26, no. 5, pp. 1474-1483, 2011.

[106] M. B. Herrera, V. Fonsato, S. Gatti et al., "Human liver stem cellderived microvesicles accelerate hepatic regeneration in hepatectomized rats," Journal of Cellular and Molecular Medicine, vol. 14, no. 6, pp. 1605-1618, 2010.

[107] R. C. Lai, F. Arslan, M. M. Lee et al., "Exosome secreted by MSC reduces myocardial ischemia/reperfusion injury," Stem Cell Research, vol. 4, no. 3, pp. 214-222, 2010.

[108] R. C. Lai, T. S. Chen, and S. K. Lim, "Mesenchymal stem cell exosome: a novel stem cell-based therapy for cardiovascular disease," Regenerative Medicine, vol. 6, no. 4, pp. 481-492, 2011.

[109] T. S. Chen, R. C. Lai, M. M. Lee, A. B. H. Choo, C. N. Lee, and S. K. Lim, "Mesenchymal stem cell secretes microparticles enriched in pre-microRNAs," Nucleic Acids Research, vol. 38, no. 1, Article ID gkp857, pp. 215-224, 2010.

[110] A. Ranghino, V. Cantaluppi, C. Grange et al., "Endothelial progenitor cell-derived microvesicles improve neovascularization in a murine model of hindlimb ischemia," International Journal of Immunopathology and Pharmacology, vol. 25, no. 1, pp. 75-85, 2012.

[111] M. Z. Ratajczak, M. Kucia, T. Jadczyk et al., "Pivotal role of paracrine effects in stem cell therapies in regenerative medicine: can we translate stem cell-secreted paracrine factors and microvesicles into better therapeutic strategies," Leukemia, vol. 26, no. 6, pp. 1166-1173, 2012.

[112] P. J. Quesenberry and J. M. Aliotta, "Cellular phenotype switching and microvesicles," Advanced Drug Delivery Reviews, vol. 62, no. 12, pp. 1141-1148, 2010.

[113] J. M. Aliotta, M. Pereira, K. W. Johnson et al., "Microvesicle entry into marrow cells mediates tissue-specific changes in mRNA by direct delivery of mRNA and induction of transcription," Experimental Hematology, vol. 38, no. 3, pp. 233-245, 2010.

[114] J. F. Renzulli II, M. Del Tatto, G. Dooner et al., "Microvesicle induction of prostate specific gene expression in normal human bone marrow cells," Journal of Urology, vol. 184, no. 5, pp. 21652171, 2010.

[115] W. A. He, F. Calore, P. Londhe, A. Canella, D. C. Guttridge, and C. M. Croce, "Microvesicles containing miRNAs promote muscle cell death in cancer cachexia via TLR7," Proceedings of the National Academy of Sciences of the United States of America, vol. 111, no. 12, pp. 4525-4529, 2014.

[116] N. Jain, A. Rossi, and G. Garcia-Manero, "Epigenetic therapy of leukemia: an update," International Journal of Biochemistry and Cell Biology, vol. 41, no. 1, pp. 72-80, 2009.

[117] J. Vojinovic, N. Damjanov, C. D’Urzo et al., "Safety and efficacy of an oral histone deacetylase inhibitor in systemic-onset juvenile idiopathic arthritis," Arthritis and Rheumatism, vol. 63, no. 5, pp. 1452-1458, 2011.

[118] J. Vojinovic and N. Damjanov, "HDAC inhibition in rheumatoid arthritis and juvenile idiopathic arthritis," Molecular Medicine, vol. 17, no. 5-6, pp. 397-403, 2011. 

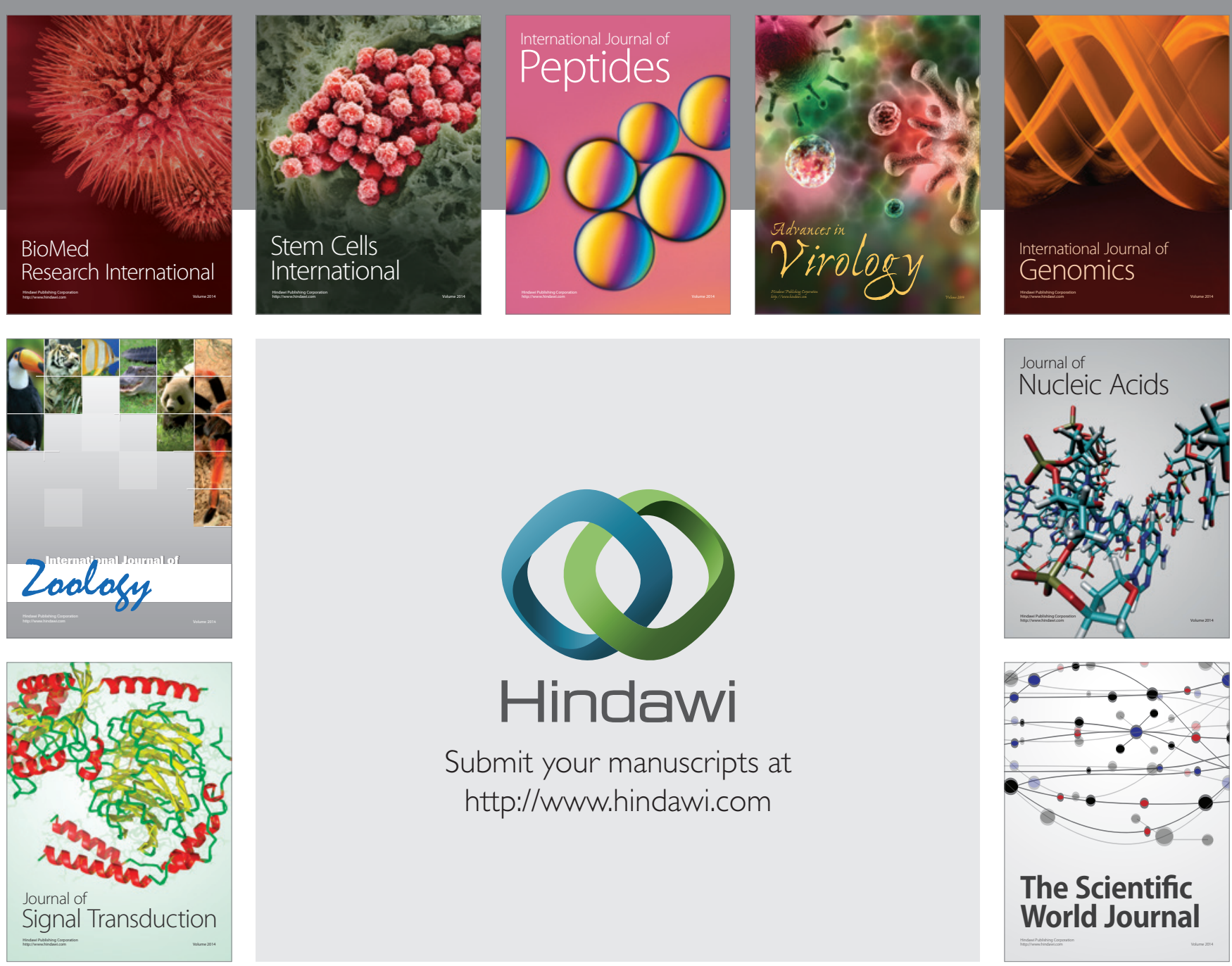

Submit your manuscripts at

http://www.hindawi.com
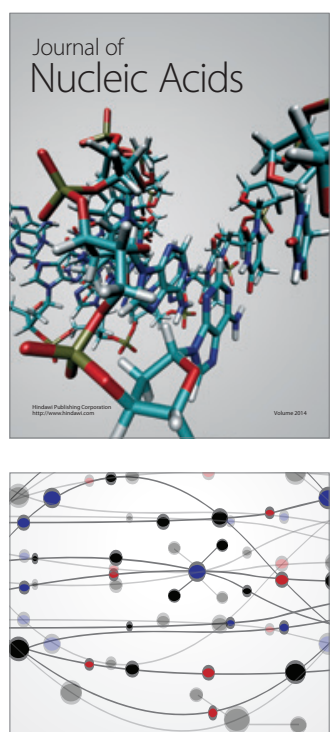

The Scientific World Journal
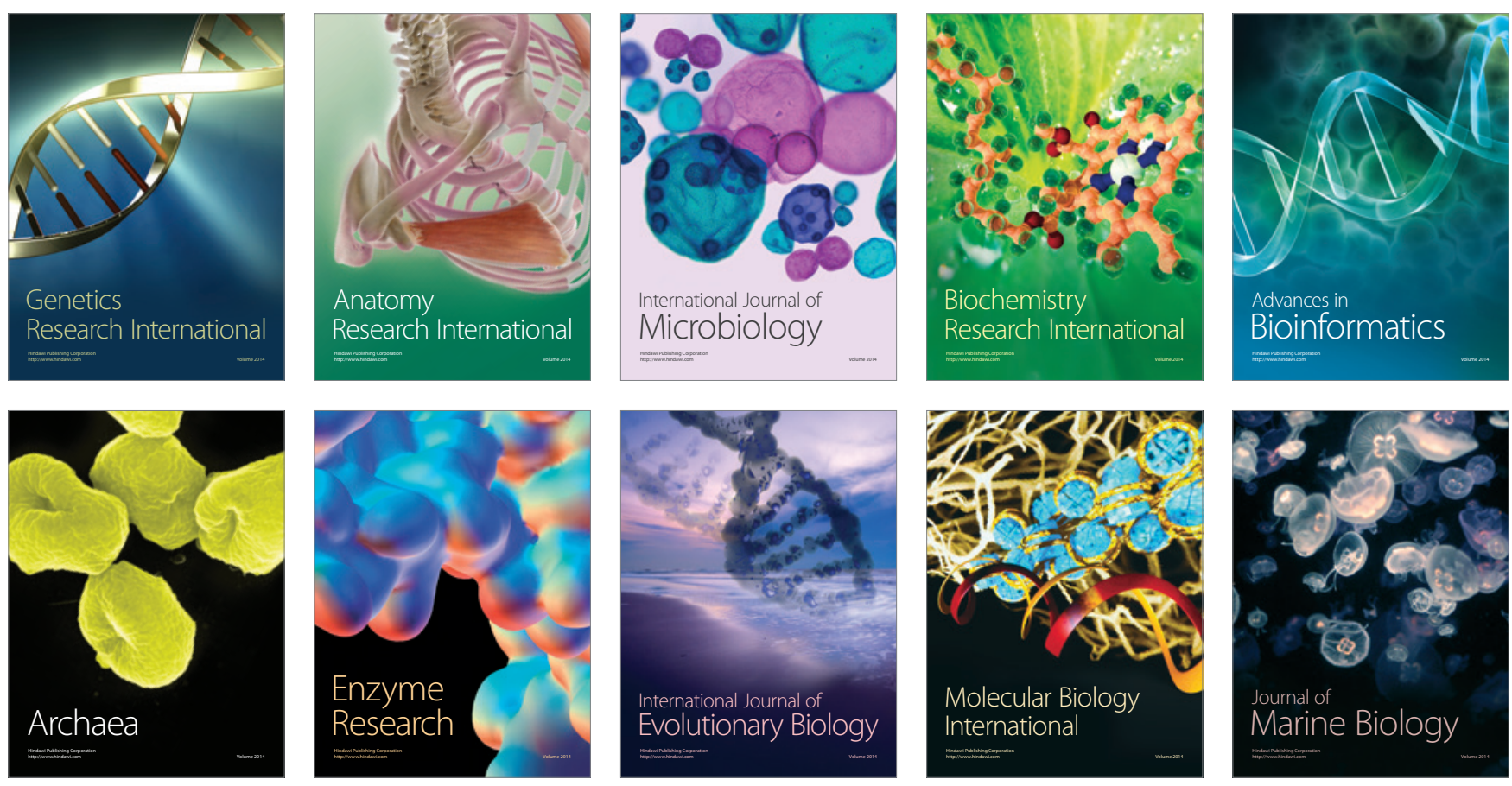